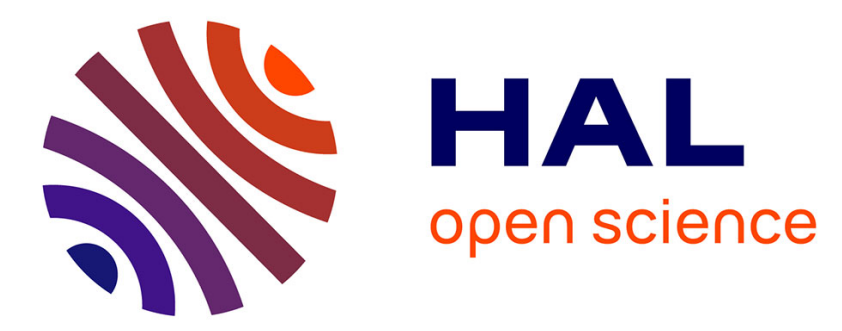

\title{
Low-strain effective Young's modulus model and validation for multi-layer vocal fold based silicone specimens with inclusions
}

M Ahmad, X Pelorson, I A Fernández, O Guasch, Annemie van Hirtum

\section{- To cite this version:}

M Ahmad, X Pelorson, I A Fernández, O Guasch, Annemie van Hirtum. Low-strain effective Young's modulus model and validation for multi-layer vocal fold based silicone specimens with inclusions. Journal of Applied Physics, 2022, 131 (5), pp.054701. 10.1063/5.0080468 . hal-03538688

\section{HAL Id: hal-03538688 https://hal.science/hal-03538688}

Submitted on 21 Jan 2022

HAL is a multi-disciplinary open access archive for the deposit and dissemination of scientific research documents, whether they are published or not. The documents may come from teaching and research institutions in France or abroad, or from public or private research centers.
L'archive ouverte pluridisciplinaire HAL, est destinée au dépôt et à la diffusion de documents scientifiques de niveau recherche, publiés ou non, émanant des établissements d'enseignement et de recherche français ou étrangers, des laboratoires publics ou privés. 
Low-strain effective Young's modulus model and validation for multi-layer vocal fold based silicone specimens with inclusions

M. Ahmad, ${ }^{1}$ X. Pelorson, ${ }^{1}$ I.A. Fernández, ${ }^{2}$ O. Guasch, ${ }^{3}$ and A. Van Hirtum ${ }^{1}$

1)Univ. Grenoble Alpes, CNRS, Grenoble INP, LEGI, 38000 Grenoble, France

${ }^{2)}$ Dep. of Materials Science and Physical Chemistry, University of Barcelona, Spain

${ }^{3)}$ Dep. of Engineering, La Salle, Universitat Ramon Llull, Spain

(*Electronic mail: annemie.vanhirtum@univ-grenoble-alpes.fr)

(Dated: 13 January 2022)

A model of the effective low-strain elastic Young's modulus of multi-layer stacked composites is proposed which is capable to account for an arbitrary stacked inclusion. Geometrical and discretisation based model results are validated against measured effective Young's moduli (from $10 \mathrm{kPa}$ up to $40 \mathrm{kPa}$ ) on 14 molded silicone specimens embedding a stiff $(298 \mathrm{kPa})$ inclusion with variable size, position and stacking. Specimens without inclusion represent the muscle, superficial and epithelium layers in a human vocal fold with Young's moduli between $4 \mathrm{kPa}$ and $65 \mathrm{kPa}$. The proposed model allows to predict the influence of a stiff inclusion, mimicking a structural abnormality or pathology somewhere within the vocal fold, on the low-strain effective Young's modulus. Quantifying the influence of an inclusion or local stiffening on the vocal fold bio-mechanics is a necessary step towards the understanding and mitigation of structural vocal folds pathologies and associated voice disorders. 


\section{INTRODUCTION}

Phonation or voiced speech sound production is due to the fluid-structure interaction (FSI) between airflow coming from the lungs along the inferior-superior direction and the vocal folds tissues enveloping the airflow within the glottis ${ }^{1}$. Although the physical mechanisms driving phonation are known ${ }^{2-4}$, systematic studies of the influence of the vocal folds structure, either normal or abnormal, on the FSI and subsequent vocal fold auto-oscillation are few ${ }^{5,6}$ as most physical studies focus on the glottal flow ${ }^{7-9}$. This is partly due to the lack of a low-cost computational model predicting the elasticity of deformable silicone vocal fold replicas mimicking, up to some extent, the multi-layer (ML) anatomical representation ${ }^{1}$ of a human vocal fold without (Fig. 1) or with structural abnormality or pathology.

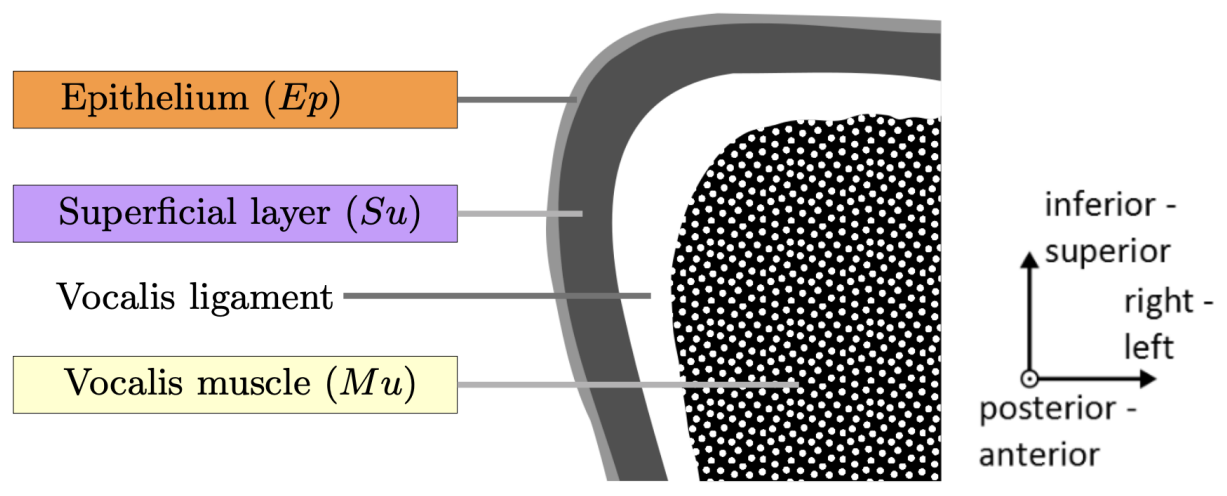

FIG. 1. Multi-layer representation of the anatomical structure of a human vocal fold in the medio-frontal plane, i.e. a coronal section.

In the linear low-strain region, the stress-strain relationship is described by Hooke's law so that the elasticity of a soft material is characterised by its low-strain or elastic Young's modulus. $\operatorname{In}^{10}$, a quasi-analytical model of the low-strain effective elastic Young's modulus $\mathcal{E}_{e f f}$ was proposed for homogenised ML silicone composites with serial $(\perp)$, parallel $(\|)$ or combined $(\perp \|)$ stacked layers with respect to the force direction, as depicted in Fig. 2. This means that composites are treated as $n$ layers which are stacked either serial or parallel to each other. The model was validated (overall accuracy of $\pm 2.4 \mathrm{kPa}$ ) against measured $\mathcal{E}_{\text {eff }}$ from uni-axial tensile tests at room temperature $21 \pm 2{ }^{\circ} \mathrm{C}$ using precision loading (PL) and a mechanical press. The validation was done on 13 bone-shaped molded silicone specimens containing two $(\perp$ or $\|)$ or three $(\perp, \|$ or $\perp \|)$ layers and $\mathcal{E}_{\text {eff }} \leq 40 \mathrm{kPa}$. The Young's modulus $\mathcal{E}$ of the molded layers was varied considering two different silicone mixtures (Thinner-Ecoflex (TE) or Thinner-Dragonskin (TD)) at different mass mixing 


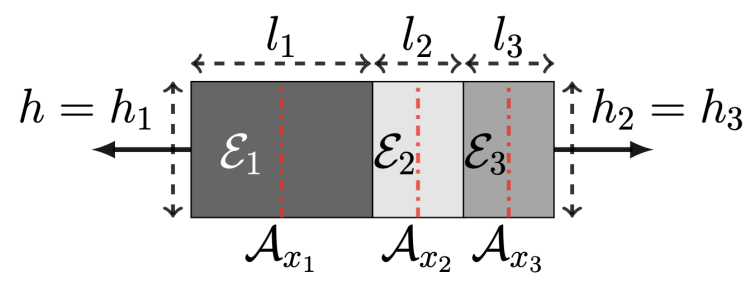

(a) serial $\perp$

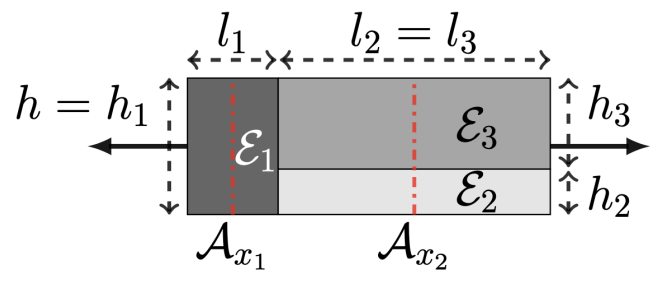

(c) combined $\perp \|$

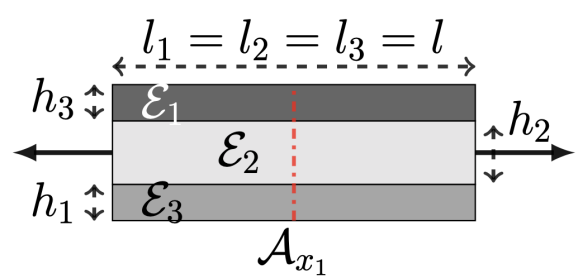

(b) parallel \|

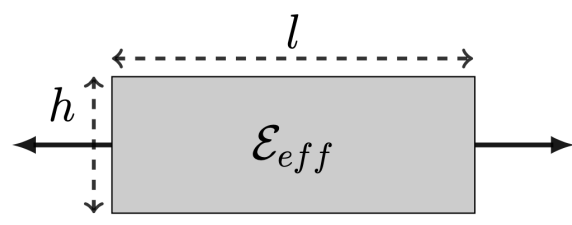

(d) homogenised

FIG. 2. Layer stacking with respect to the force direction (black arrows) for three-layer $(n=3)$ composites with height $h$ and length $l$ : a) serial $(\perp)$, b) parallel $(\|)$, c) combined $(\perp \|)$ and d) sought homogenised composite with effective Young's modulus $\mathcal{E}_{\text {eff }}$. Layers Young's moduli $\mathcal{E}_{i}$ and dimensions (height $h_{i}$, length $l_{i}$ ) are indicated with $i=1 \ldots n$. Positions of midway cross-section area measurements $\mathcal{A}_{x_{s}}$ are indicated (dash-dotted line) for each serial stacked equivalent layer $s=1 \ldots k$ so that in a) $k=3, \mathrm{~b}$ ) $k=1$ and c) $k=2$.

ratios $\mathcal{M}=r_{T}: r_{E(D)}$ with constant $r_{E(D)}=2$. Silicone mixtures, mixing ratios $\mathcal{M}$ and measured $\mathcal{E}$ commonly used to represent the muscle $(\mathrm{Mu})$, superficial $(\mathrm{Su})$ and epithelium (Ep) layer in ML silicone vocal fold replicas are given in Table $\mathrm{I}^{10-18}$. The measured Young's modulus $\mathcal{E}$ of molded layers varies between $4 \mathrm{kPa}$ and $65 \mathrm{kPa}$, which corresponds to the range associated with anatomical layers in a normal adult male human vocal fold ${ }^{19-25}$.

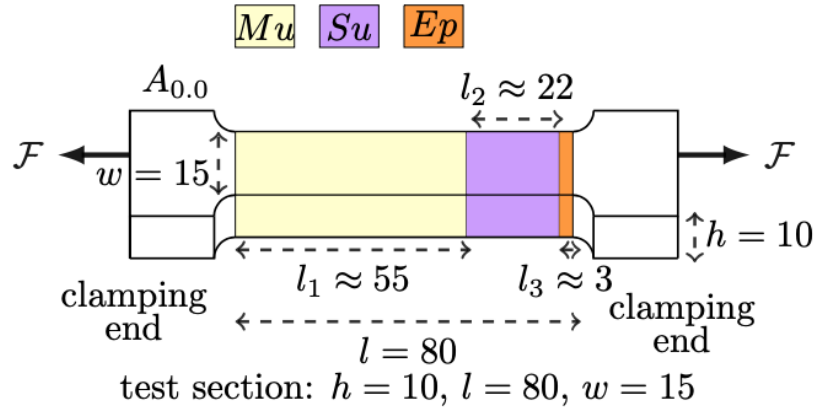

FIG. 3. Molded bone-shaped three-layer reference specimen $A_{0.0}$ with serial stacking $(\perp)$ without inclusion (dimensions in $\mathrm{mm}$ ). The force $\mathcal{F}$ direction during uni-axial tension testing is shown (black arrows). 
In the current work, it is aimed to propose a model approach predicting $\mathcal{E}_{\text {eff }}$ for multi-layered specimen containing a stiff inclusion. The model outcome is validated against uni-axial tensile test measurements (using precision loading ${ }^{10}$ ) on molded bone-shaped composite specimens. All experiments are done at room temperature $21 \pm 2{ }^{\circ} \mathrm{C}$. Specimens are obtained from a three-layer reference specimen (labelled $A_{0.0}$ ) to which a stiffer silicone inclusion (In) is inserted with constant elasticity $\mathcal{E}^{I n}$ given in Table I. The reference specimen without inclusion is depicted in Fig. 3. It consists of three serial $(\perp)$ stacked layers with a composition similar to the muscle, superficial and epithelium layer indicated in Table I. The length $l_{i}$ of each layer $i=1 \ldots n$ in the force $(\mathcal{F})$ direction, with $n=3$ serial stacked layers, is set so that the volume ratios for the muscle, the superficial and the epithelium layer with respect to the test section's volume match the volume ratios of a three-layer silicone vocal fold replica (MRI-replica $\left.{ }^{14,16-18}\right)$, i.e. $69 \%(\mathrm{Mu}, i=1), 27 \%(\mathrm{Su}$, $i=2)$ and $4 \%(\mathrm{Ep}, i=3)$ respectively. The size, the position or the orientation of the inclusions are varied resulting in complex ML composites. From the ratio $\mathcal{E}^{I n} / \mathcal{E}$ in Table I is seen that $\mathcal{E}^{\operatorname{In}}$ is about 4.5 up to 75 times greater than $\mathcal{E}$ associated with the layers in $A_{0.0}$. The presence of a stiffer portion somewhere within the vocal fold structure is commonly reported in case of a vocal fold abnormality or pathology ${ }^{1,26-28}$. Consequently, a validated model is sought for ML composite specimens embedding a stiffer inclusion, for which besides the Young's modulus also the size, position and stacking orientation is varied. This will contribute to the systematic prediction and understanding of the effect of an inclusion on the low-strain elasticity of silicone ML vocal folds replicas mimicking a local vocal fold structural abnormality or pathology.

Concretely, six specimen types (resulting in 15 molded specimens) are experimentally assessed in order to complete the model validation with respect to the main inclusion characteristics: position,

TABLE I. Molded layer properties: mixture (Mix) TE (Thinner-Ecoflex) or TD (Thinner-Dragonskin), mass mixing ratio $\mathcal{M}$, measured Young's modulus $\mathcal{E}$, ratio between $\mathcal{E}$ and the value of the inclusion $\mathcal{E}^{\text {In }}$.

\begin{tabular}{l|cccc}
\hline Molded layer & Mix & $\mathcal{M}[-]$ & $\mathcal{E}[\mathrm{kPa}]$ & $\mathcal{E}^{I n} / \mathcal{E}[-]$ \\
\hline Muscle (Mu) & TE & $1: 2$ & 23 & 13.0 \\
Superficial (Su) & TE & $4: 2$ & 4 & 74.5 \\
Epithelium (Ep) & TD & $1: 2$ & 65 & 4.6 \\
Inclusion (In) & TD & $0: 2$ & $298^{\star}$ & 1.0 \\
\hline
\end{tabular}

$\star \mathcal{E}$ of the inclusion material is denoted $\mathcal{E}^{I n}$. 
size (length and height) and shape. This validation is pertinent to the representation with siliconebased vocal fold replicas of vocal fold pathologies characterised by an arbitrary-shaped inclusion of any size embedded somewhere within a single or multiple vocal fold layers. The specimen types with inclusions are presented in section II. Molded specimens are motivated. Next, the measurement of low-strain effective Young's moduli for the molded specimens is detailed in section III. The analytical model approach for the effective Young's modulus of ML composite specimens with an inclusion and serial, parallel or combined stacking is outlined in section IV. A discretisation and geometrical based model approach for arbitrary stacked layers is proposed. Measured and modelled effective Young's moduli for different inclusions are discussed in section V. The conclusion is formulated in section VI.

\section{MOLDED SPECIMENS WITH INCLUSION}

Fig. 4 schematically illustrates the different positions of beam-shaped inclusions with varying height $h_{\text {in }}$ and length $l_{\text {in }}$ inserted (striped region) in the test section of the reference specimen $A_{0.0}$ with serial layer stacking $(\perp)$ depicted in Fig. 3. All inclusions have constant width $w_{\text {in }}=15 \mathrm{~mm}$, which matches the width of the test section ( $w_{\text {in }}=w$ with $w=15 \mathrm{~mm}$ ) as illustrated for two specimens with inclusions in Fig. 5. Thus, the inclusion size is fully characterised by its height $h_{\text {in }}$ and its length $l_{\text {in }}$ and its position is fully defined by the side views provided in Fig. 4. Six different ML composite specimen types - A, B, C, D, E and F - are considered based on the position and size of the inclusion. Concretely, the size, in terms of height $h_{\text {in }}$ and length $l_{i n}$, of experimentally assessed inclusions is summarized in Table II:

(A) specimens $A_{h_{\text {in }} / h}$ (Fig. 4(a)) are obtained for 5 inclusions with constant length $l_{\text {in }}=l_{2}$ and varying height $0.1 \leq h_{\text {in }} / h \leq 1$ placed at the side of the superficial layer ( $\left.\mathrm{Su}, i=2\right)$ of $A_{0.0}$. In general, these specimens have four layers $(n=4)$ with combined stacking $(\perp \|)$ as the inclusion in the superficial layer results in two adjacent layers with parallel stacking. This is illustrated for $A_{0.3}\left(h_{i n} / h=0.3\right)$ in Fig. 5(a). In the extreme case, that the inclusion replaces the superficial layer $\left(h_{i n} / h=1.0\right)$, a three-layer $(n=3)$ specimen $A_{1.0}$ with serial stacking $(\perp)$ is obtained as illustrated in Fig. 5(b).

(B) specimens $B_{h_{i n} / h}$ (Fig. 4(b)) are obtained for 3 of the 5 inclusions with constant length $l_{\text {in }}=l_{2}$ and varying height $0.1 \leq h_{\text {in }} / h \leq 0.6$ considered in type A, but now translated (in 
Low-strain inclusion

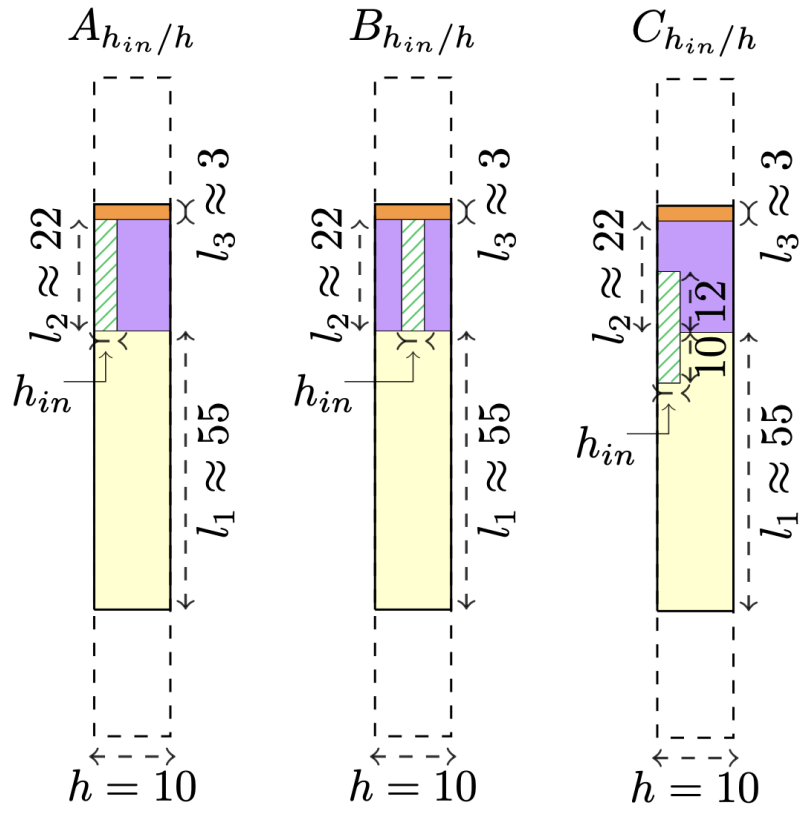
(a) type A
(b) type B
(c) type $\mathrm{C}$
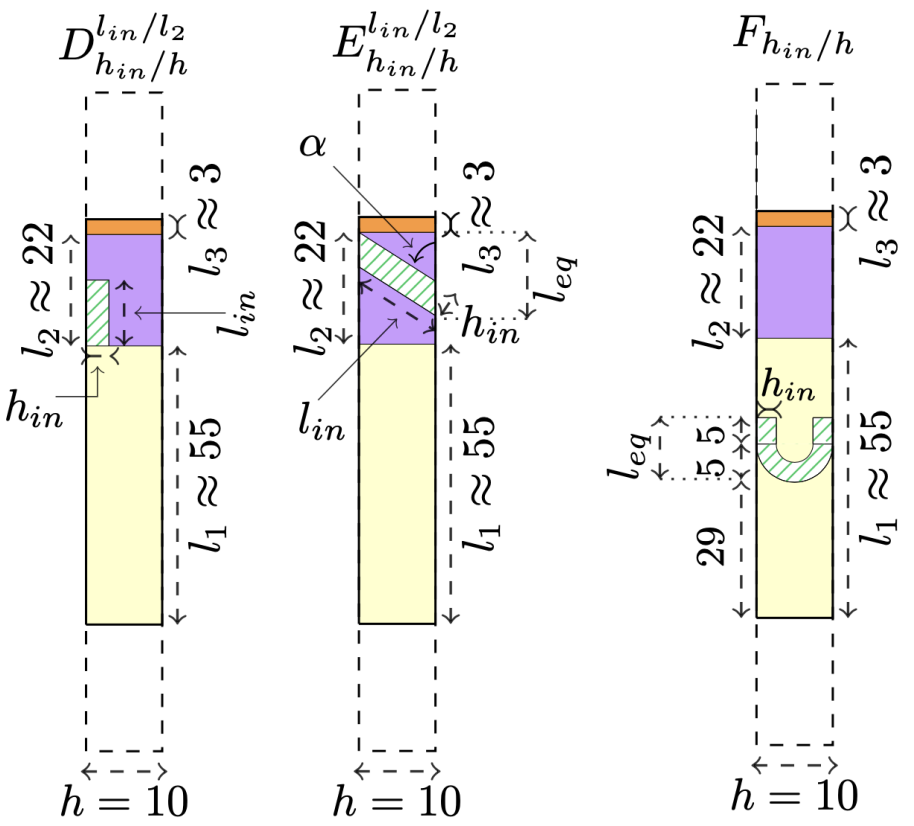

(d) type D

(e) type E

(f) type F

FIG. 4. Side view of molded ML composite types with inclusions (striped region) of size $h_{\text {in }}, l_{\text {in }}$ and constant width $w_{i n}=15 \mathrm{~mm}$ (dimensions in $\mathrm{mm}$ ). In a,b,c,f) $l_{i n}=l_{2}$. In d,e) $l_{i n}<l_{2}$. The clamping ends are dashed.

the transverse direction) to the center of the superficial layer ( $\mathrm{Su}, i=2)$ in $A_{0.0}$. Comparing the elasticity of $A_{h_{i n} / h}$ and $B_{h_{i n} / h}$ allows to assess the influence of the transverse inclusion position (side versus center) within the superficial layer of $A_{0.0}$. These specimens are com- 
posed of five layers $(n=5)$ with combined stacking $(\perp \|)$ as the inclusion in the superficial layer results in three adjacent parallel stacked layers.

(C) specimen $C_{h_{\text {in }} / h}$ (Fig. 4(c)) is obtained using the inclusion with constant length $l_{i n}=l_{2}$ and height $h_{\text {in }} / h=0.8$ positioned in both the superficial and the muscle layer of $A_{0.0}$. This specimen can be considered as a seven-layer $(n=7)$ specimen with combined stacking $(\perp \|)$ as the inclusion has parallel stacking with respect to the adjacent portions of the superficial layer and the muscle layer.

(D) specimens $D_{h_{\text {in }} / h}^{l_{\text {in }} / l_{2}}$ (Fig. 4(d)) are obtained for 5 inclusions with varying length $0.2 \leq l_{\text {in }} / l_{2} \leq$ 0.6 and varying height $0.3 \leq h_{\text {in }} / h \leq 0.8$ placed at the side of the superficial layer and at the interface with the muscle layer. These specimens are considered to have five layers $(n=5)$ with combined stacking $(\perp \|)$ as the inclusion has a parallel stacking with respect to the adjacent portion of the superficial layer with the same height. Thus, the inclusion length is reduced so that comparing type $\mathrm{A}$ and type $\mathrm{D}$ allows one to assess the influence of the

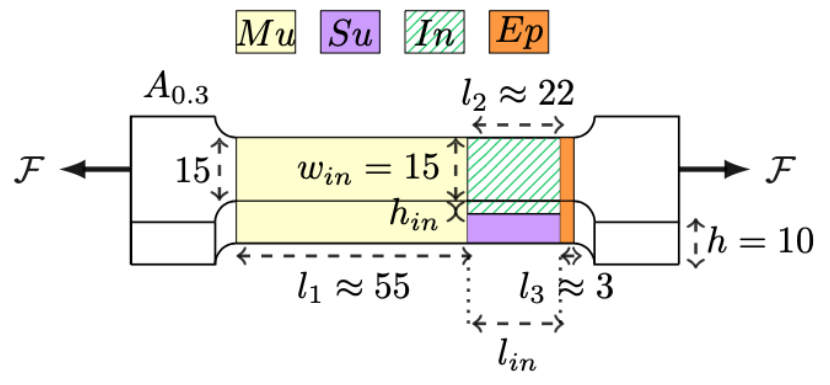

(a) $A_{0.3}$

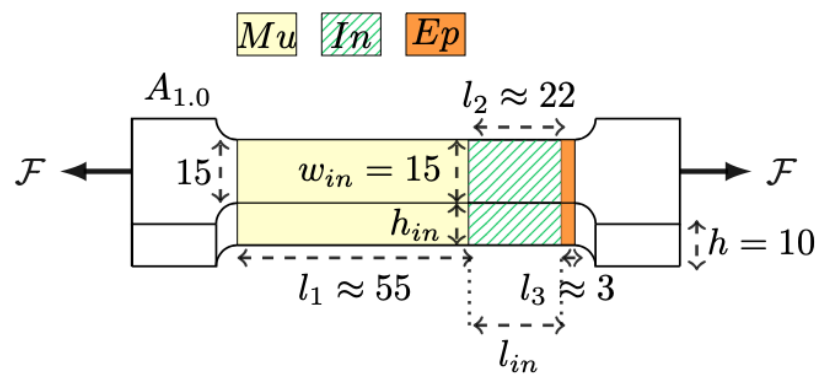

(b) $A_{1.0}$

FIG. 5. Molded ML bone-shaped specimens (dimensions in $\mathrm{mm}$ ) with inclusion (In) inserted in the superficial ( $\mathrm{Su}$ ) layer of the reference specimen $A_{0.0}$ depicted in Fig. 3: a) four-layer specimen $A_{0.3}$ with combined stacking $(\perp \|)$, b) three-layer specimen $A_{1.0}$ with serial stacking $(\perp)$. The force $\mathcal{F}$ direction during uni-axial tension testing is shown (black arrows). 
inclusion length.

(E) specimen $E_{h_{\text {in }} / h}^{l_{\text {in }} / l_{2}}$ (Fig. 4(e)) is obtained by inclining the inclusion with angle $\alpha\left(\alpha=46^{\circ}\right.$ is experimentally assessed). The inclined inclusion is placed in the superficial layer at the interface with the epithelium layer. Due to the inclination, the stacking in the superficial layer is arbitrary $(\mathrm{Arb})$ and it is neither serial $(\perp)$ nor parallel $(\|)$ with respect to the adjacent superficial layer portion. The equivalent length $l_{e q}$ of the inclusion corresponds to its length in the force direction and $l_{e q}<l_{\text {in }}$ due to the inclination.

(F) specimen $F_{h_{i n} / h}$ (Fig. 4(f)) is obtained by bending the inclusion with length $l_{i n}=l_{2}$ and placing it in the muscle layer. As for type E, the stacking orientation within the muscle layer is arbitrary (Arb) since the bent portion of the inclusion is stacked neither serial $(\perp)$ nor parallel $(\|)$ with respect to the adjacent muscle layer portion. Again, the equivalent length $l_{e q}$ of the inclusion corresponds to its length in the force direction and $l_{e q}<l_{\text {in }}$ due to the bending.

A total of 15 silicone specimens are molded following the mixture procedure outlined in ${ }^{10,29}$.

TABLE II. Inclusions geometry (constant width $w_{\text {in }}=15 \mathrm{~mm}$ ): $h_{\text {in }}, l_{\text {in }}$, ratios $h_{\text {in }} / h$ and $l_{\text {in }} / l_{2}$ with $h \approx$ $10 \mathrm{~mm}$ and $l_{2} \approx 22 \mathrm{~mm}$. ML molded specimens with these inclusions and their layer stacking: serial $(\perp)$, combined $(\perp \|)$ and arbitrary (Arb). Reference specimen $A_{0.0}$ corresponds to $h_{\text {in }} / h=0$.

\begin{tabular}{|c|c|c|c|c|c|c|}
\hline \multirow{2}{*}{\multicolumn{2}{|c|}{$\begin{array}{l}\text { Inclusion geometry } \\
h_{\text {in }}[\mathrm{mm}] l_{\text {in }}[\mathrm{mm}]\end{array}$}} & \multicolumn{2}{|c|}{ Dimension ratios } & \multicolumn{3}{|c|}{ Specimens \& stacking } \\
\hline & & $h_{\text {in }} / h[-$ & in $/ l_{2}[-]$ & $(\perp)$ & $(\perp \|)$ & Arb \\
\hline 0.0 & - & 0.0 & 0.0 & $A_{0.0}$ & & \\
\hline 1.3 & 22.0 & 0.1 & 1.0 & & $A_{0.1} B_{0.1}$ & \\
\hline 2.6 & 22.0 & 0.3 & 1.0 & & $A_{0.3} B_{0.3}$ & $F_{0.3}$ \\
\hline 5.8 & 22.0 & 0.6 & 1.0 & & $A_{0.6} B_{0.6}$ & \\
\hline 7.8 & 22.0 & 0.8 & 1.0 & & $A_{0.8} C_{0.8}$ & \\
\hline 10.0 & 22.0 & 1.0 & 1.0 & $A_{1.0}$ & & \\
\hline 2.6 & 5.0 & 0.3 & 0.2 & & $D_{0.3}^{0.2}$ & \\
\hline 7.8 & 5.0 & 0.8 & 0.2 & & $D_{0.8}^{0.2}$ & \\
\hline 4.6 & 13.4 & 0.5 & 0.6 & & $D_{0.5}^{0.6}$ & $E_{0.5}^{0.6}$ \\
\hline
\end{tabular}


The size and position of the inclusion and the resulting ML specimen type (Fig. 4) and associated layer stacking - serial $(\perp)$, combined $(\perp \|)$ or arbitrary (Arb) - is summarised in Table II. Firstly, inclusions are molded using horizontal 3D-printed specimen molds (Stratasys ABS-P430, accuracy $0.33 \mathrm{~mm}$ ), which are filled with the inclusion silicone mixture indicated in Table I up to each inclusion's height $h_{\text {in }}$ whereas their width is determined by the width of the mold so that $w_{i n}=15 \mathrm{~mm}$. The molded inclusion sheets are then cut to match the desired inclusion length $l_{i n}$. Next, vertical 3D-printed specimen molds are used to build the ML specimens layer-by-layer with the appropriate silicone mixture detailed in Table I. Inclusions are inserted during the molding process so that they are fully embedded. The length of each molded layer is measured with a laser transceiver (Panasonic HL-G112-A-C5, wavelength $655 \mathrm{~nm}$, accuracy $8 \mu \mathrm{m}$ ) whereas the inclination angle is derived from the spatial position of the inclined mold (accuracy $0.1 \mathrm{~mm}$ ) using trigonometry. The overall molding accuracy for all 45 molded layers with lengths $l_{1} \approx 55 \mathrm{~mm}$ (muscle), $l_{2} \approx 22 \mathrm{~mm}$ (superficial) and $l_{3} \approx 3 \mathrm{~mm}$ (epithelium) results in a mean and a standard deviation of $0.1 \pm 0.8 \mathrm{~mm}$, which is within the molding accuracy of $\pm 1.5 \mathrm{~mm}$ previously reported ${ }^{10}$.

\section{UNI-AXIAL TENSION TESTS AND $\mathcal{E}_{e f f}$ ESTIMATION}

\section{A. Uni-axial tension test data on molded specimen}

In order to experimentally estimate the linear or low-strain effective Young's moduli $\mathcal{E}_{\text {eff }}$ of the 15 molded composite specimens, uni-axial tension tests using precision loading (PL) are performed $^{10}$. Firstly, the tested specimen is placed vertically and fixed from one clamping end. Then, a weight of mass $m$ (Vastar $500 \mathrm{G}$ X $0.01 \mathrm{G}$, accuracy $0.01 \mathrm{~g}$ ) is added to the other clamping end in order to control the force increment. The weight is increased at a single rate for each specimen. Overall, i.e. considering all specimens, the weight is incremented with 10.9 (6.9) $\mathrm{g}$ (mean (and standard deviation)). Total added weight ranges from $46.8 \mathrm{~g}$ to $425.9 \mathrm{~g}$ resulting in a total applied loading force $\mathcal{F}$ ranging from $0.5 \mathrm{~N}$ to $4.2 \mathrm{~N}$. The applied force $\mathcal{F}$ causes an elongation $\Delta l_{s}$ of each equivalent serial stacked layer with initial length $l_{s}$. The total elongation $\Delta l=\sum_{s=1}^{k} \Delta l_{s}$ is then measured at every added weight increment with an accuracy of $0.05 \mathrm{~mm}$ where $k$ indicates the number of equivalent serial stacked layers. Total elongation measured for all specimens ranges from $23.0 \mathrm{~mm}$ up to $131.0 \mathrm{~mm}$. From these measurements, force-elongation relationship $\mathcal{F}(\Delta l)$ 
can be obtained. Examples of two force-elongation diagrams for two different specimens $A_{0.0}$ and $A_{0.8}$ are plotted in Fig. 6(a). Additionally, the midway cross-sectional area of each equivalent serial stacked layer $\mathcal{A}_{x_{s}}$ perpendicular to the forcing direction is measured with an accuracy of $0.02 \mathrm{~mm}$ as illustrated in Fig. 2. For each specimen, cross-sectional areas $\mathcal{A}_{x_{s}}$ are measured at a constant weight increment amounting to $26.7 \pm 15.9 \mathrm{~g}$ for all specimens, which corresponds to an elongation increment of $10.6 \pm 4.5 \mathrm{~mm}$. The specimen cross-sectional area $\mathcal{A}$ is then calculated from the arithmetic mean of its measured cross-sectional areas weighted by their respective lengths as

$$
\mathcal{A}=\frac{\sum_{s=1}^{k}\left(l_{s}+\Delta l_{s}\right) \mathcal{A}_{x_{s}}}{l+\Delta l} .
$$

A quadratic fit (coefficient of determination $R^{2} \geq 99 \%$ ) to the area-elongation data $\mathcal{A}(\Delta l)$ is then used in order to have a continuous approximation of the area-elongation relationship $\mathcal{A}^{q}(\Delta l)$ for each specimen. An example of resulting data points $\mathcal{A}(\Delta l)$ and their continuous fit $\mathcal{A}^{q}(\Delta l)$ for two different specimens $A_{0.0}$ and $A_{1.0}$ is plotted in Fig. 6(b).

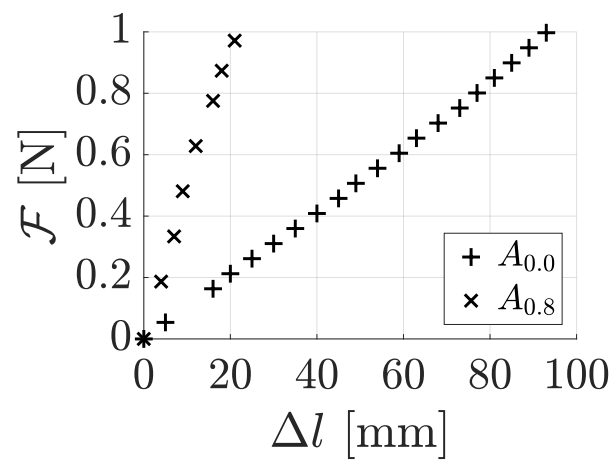

(a) $\mathcal{F}(\Delta l)$

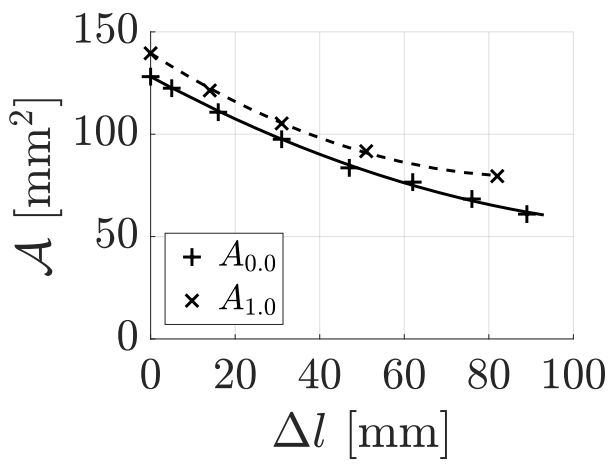

(b) $\mathcal{A}(\Delta l)$

FIG. 6. Examples of uni-axial tension testing data: a) force-elongation data $\mathcal{F}(\Delta l)$ for specimens $A_{0.0}$ and $A_{0.8}$ with $m \leq 102 \mathrm{~g}$ and $m \leq 231 \mathrm{~g}$, respectively, b) area-elongation data $\mathcal{A}(\Delta l)$ and quadratic fits $\mathcal{A}^{q}(\Delta l)$ with $R^{2}=99 \%$ (lines) for specimens $A_{0.0}$ and $A_{1.0}$.

\section{B. Experimental $\mathcal{E}_{e f f}$ estimation from tensile test data}

In order to estimate the linear low-strain effective Young's modulus $\mathcal{E}_{\text {eff }}$ for each specimen, stress-strain curves $\sigma_{t}\left(\varepsilon_{t}\right)$ are firstly calculated from the measured instantaneous elongation $\Delta l$ 
and associated force $\mathcal{F}(\Delta l)$ and area $\mathcal{A}^{q}(\Delta l)$. The true stress $\sigma_{t}$ and true strain $\varepsilon_{t}$ are then given as

$$
\begin{gathered}
\sigma_{t}=\frac{\mathcal{F}}{\mathcal{A}^{q}}, \\
\varepsilon_{t}=\ln \left(\frac{l+\Delta l}{l}\right) .
\end{gathered}
$$

The sought $\mathcal{E}_{\text {eff }}$ is obtained by fitting the low-strain region of the stress-strain curves to a linear model whose slope equals $\mathcal{E}_{e f f}$, as the relationship between stress and low-strain is governed by Hooke's law

$$
\mathcal{E}_{e f f}=\frac{\sigma_{t}}{\varepsilon_{t}} .
$$

For each specimen, the low-strain region is determined as the range for which $R^{2}$ is maximum, where $R^{2} \geq 98 \%$ expresses the goodness of fitting a linear model to the stress-strain curve with a lower bound at $\varepsilon_{t}=0$. For all specimens tested, the mean (and standard deviation) of the upper bound of the low-strain region is $\varepsilon_{t}=0.26(0.02)$, which corresponds to a total elongation of $24.5(1.7) \mathrm{mm}$. Examples of fitting a linear model to a low-strain region are plotted in Fig. 7 for three different specimens. Fig. 7(a) shows the different fits, and hence slopes $\mathcal{E}_{\text {eff }}$, obtained for two specimens $A_{0.0}$ and $A_{0.6}$, without and with an inclusion, respectively. Fig. 7(b) shows similar fits, and hence slopes $\mathcal{E}_{e f f}$, obtained for two specimens $A_{0.6}$ and $B_{0.6}$ with the same inclusion ( $h_{\text {in }} / h=0.6$ and $\left.l_{\text {in }} / l_{2}=1\right)$ but positioned at either the side $\left(A_{0.6}\right)$ or the center $\left(B_{0.6}\right)$ of the superficial layer in $A_{0.0}$.

\section{EFFECTIVE YOUNG'S MODULUS $\widehat{\mathcal{E}}_{e f f}$ MODEL}

\section{A. Serial, parallel and combined layer stacking}

For the composite specimens in Table II and in Fig. 4 with serial $(\perp)$ or combined $(\perp \|)$ stacked layers - i.e. of type A (Fig. 4(a)), type B (Fig. 4(b)), type C (Fig. 4(c)) or type D (Fig. 4(d)) - the

effective Young's modulus $\widehat{\mathcal{E}}_{\text {eff }}$ is modelled considering an equivalent homogeneous composite (e.g. in Fig. 2(d)) as outlined in ${ }^{10}$ for serial, parallel or combined stacked layers.

For $k$ serial $(\perp)$ stacked layers (e.g. in Fig. 2(a)), the Reuss hypothesis of homogeneous stress ${ }^{30}$ between the stress in the equivalent homogeneous composite $\sigma$ and the stress $\sigma_{i=1 \ldots k}$ in each layer is applied so that $\sigma=\sigma_{i=1 \ldots k}$. The effective Young's modulus of the equivalent homogeneous 


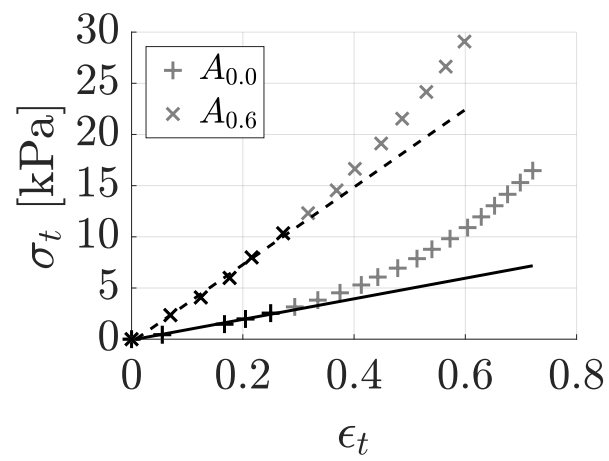

(a) Size

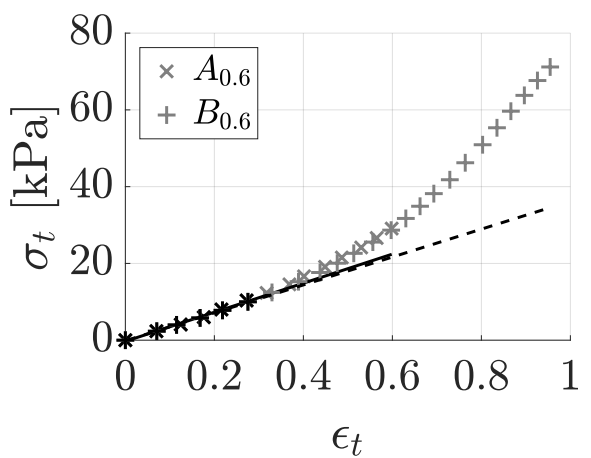

(b) Position

FIG. 7. Experimental stress-strain curves $\sigma_{t}\left(\varepsilon_{t}\right)$ for three specimens and linear fits (lines) to the low-strain region $\left(R^{2} \geq 98 \%\right)$ with slope $\mathcal{E}_{\text {eff }}$ : a) specimens $A_{0.0}$ and $A_{0.6}$ (low-strain region $\varepsilon_{t} \leq 0.25$ and $\varepsilon_{t} \leq 0.27$ ), b) specimens $A_{0.6}$ and $B_{0.6}$ (low-strain region $\varepsilon_{t} \leq 0.27$ ).

composite is then obtained as the harmonic mean of the layers Young's moduli $\mathcal{E}_{i}$ weighted with their lengths $l_{i}$ so that

$$
\widehat{\mathcal{E}}_{e f f}^{\perp}=\frac{\sum_{i=1}^{k} l_{i}}{\sum_{i=1}^{k}\left(\frac{l_{i}}{\mathcal{E}_{i}}\right)}
$$

with $l=\sum_{i=1}^{k} l_{i}$ being the length of the equivalent homogeneous composite. Eq. (5) is independent of the layer order in the serial stack.

For $k$ parallel (\|) stacked layers (e.g. in Fig. 2(b)), the Voigt hypothesis of homogeneous strain ${ }^{31}$ between the strain in the equivalent homogeneous composite $\varepsilon$ and the strain $\varepsilon_{i=1 \ldots k}$ in each layer is applied so that $\varepsilon=\varepsilon_{i=1 \ldots k}$. The effective Young's modulus of the equivalent homogeneous composite is then obtained as the arithmetic mean of the layers Young's moduli $\mathcal{E}_{i}$ weighted with their heights $h_{i}$, so that

$$
\widehat{\mathcal{E}}_{\text {eff }}^{\|}=\frac{\sum_{i=1}^{k} h_{i} \mathcal{E}_{i}}{\sum_{i=1}^{k} h_{i}}
$$

with $h=\sum_{i=1}^{k} h_{i}$ the height of the equivalent homogeneous composite. Eq. (6) is independent of the layer order in the parallel stack. 


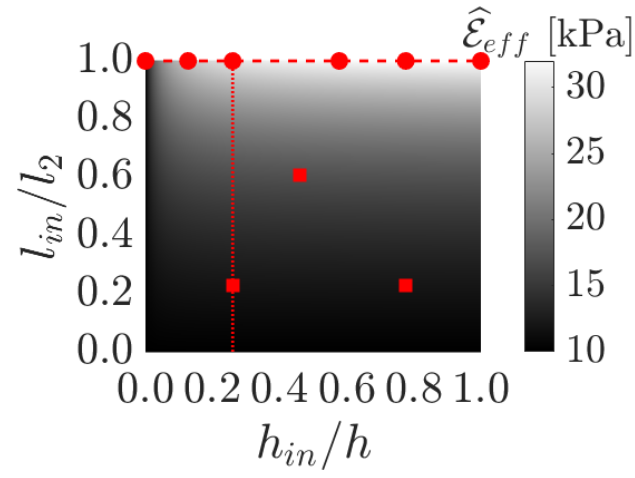

(a) varied $l_{\text {in }} / l_{2}$ and $h_{\text {in }} / h$

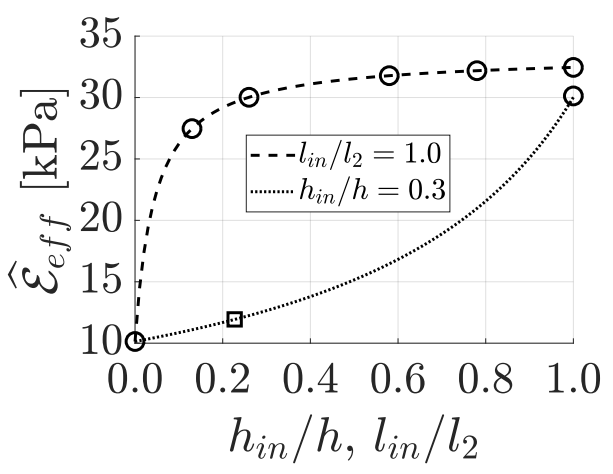

(b) constant $l_{\text {in }} / l_{2}$ or $h_{\text {in }} / h$

FIG. 8. Influence of the height ratio $0 \leq h_{\text {in }} / h \leq 1$ and length ratio $0 \leq l_{\text {in }} / l_{2} \leq 1$ of an inclusion in the superficial layer on modelled $\widehat{\mathcal{E}}_{\text {eff }}$. Values for molded specimens $(\bigcirc, \square)$ are shown: a) specimens type A (or B) $(\bigcirc)$ and type $\mathrm{D}(\square)$, constant length ratio $l_{\text {in }} / l_{2}=1.0$ (horizontal dashed line) and constant height ratio $h_{\text {in }} / h=0.3$ (dashed vertical line), b) detail for these constant length and height ratios.

Specimens with combined $(\perp \|)$ stacking (e.g. in Fig. 2(c)) contain both serial and parallel stacked layers. The effective Young's modulus of the equivalent homogeneous composite $\mathcal{E}_{\text {eff }}^{\perp \|}$ of specimens with combined stacking is then modelled using first Eq. (6) to homogenise parallel stacked layers followed by applying Eq. (5) to the remaining equivalent stack of serial layers. As layers with high $\mathcal{E}_{i}$ contribute more to the arithmetic mean (Eq. (5) in $\widehat{\mathcal{E}}_{\text {eff }}^{\|}$) than to the harmonic mean (Eq. (6) in $\widehat{\mathcal{E}}_{e f f}^{\perp}$ ) the subsequent steps in the homogenisation procedure of the layer stack (from combined stacking to equivalent serial stack to homogeneous equivalent) can not be permuted.

Inclusions associated with type $\mathrm{A}$, type $\mathrm{B}$, type $\mathrm{C}$ and type $\mathrm{D}$ in Fig. 4 result in serial $(\perp)$ or combined $(\perp \|)$ stacked layers as summarised in Table II. The effective Young's modulus $\widehat{\mathcal{E}}_{\text {eff }}$ of the molded specimens from these types are thus modelled as $\widehat{\mathcal{E}}_{\text {eff }}=\widehat{\mathcal{E}}_{\text {eff }}^{\perp}$ or $\widehat{\mathcal{E}}_{\text {eff }}=\widehat{\mathcal{E}}_{\text {eff }}^{\perp \|}$. Following this model approach, shifting the same inclusion from the side to the center of the superficial layer does not affect the model outcome. Thus $\widehat{\mathcal{E}}_{\text {eff }}$ for type A and type B specimens containing an inclusion with the same height ratio $h_{i n} / h$ have equal value. Indeed as $\mathcal{E}_{i}$ are similar, modelled $\widehat{\mathcal{E}}_{\text {eff }}$ depend solely on the height ratio $0 \leq h_{\text {in }} / h \leq 1$ and length ratio $0 \leq l_{\text {in }} / l_{2} \leq 1$ of the inclusion and not on its position. The influence of $h_{i n} / h$ and $l_{\text {in }} / l_{2}$ on $\widehat{\mathcal{E}}_{\text {eff }}$ is illustrated in Fig. 8. Model values for molded specimens are indicated (symbols). Extending the size of the inclusion in the superficial layer increases modelled $\widehat{\mathcal{E}}_{\text {eff }}$ from $\widehat{\mathcal{E}}_{\text {eff }}=10 \mathrm{kPa}$ for the reference specimen $A_{0.0}$ 
without inclusion up to about $\widehat{\mathcal{E}}_{\text {eff }}=33 \mathrm{kPa}$ for specimen $A_{1.0}$, for which the inclusion occupies the entire superficial layer. Nevertheless, Fig. 8(a) shows that $\widehat{\mathcal{E}}_{\text {eff }}$ increases more rapidly with $l_{\text {in }} / l_{2}$ than with $h_{\text {in }} / h$. In particular, this is the case for $h_{\text {in }} / h \geq 0.2$. This is due to the arithmetic mean associated with parallel stacked layers in Eq. (6), which tends to mask low $\mathcal{E}_{i}$ in favor of the large $\mathcal{E}_{i}$ of the inclusion (Table I) which is not the case for the harmonic mean associated with serial stacking in Eq. (5). Therefore, it is mainly the length ratio $l_{\text {in }} / l_{2}$ of the inclusion in the superficial layer what affects the mean value $\widehat{\mathcal{E}}_{\text {eff }}^{\|}$of the equivalent homogenised superficial layer and hence $\widehat{\mathcal{E}}_{\text {eff }}$ of the homogenised specimen. This is further illustrated for $l_{\text {in }} / l_{2}=1$ in Fig. 8(b) as an increase of $h_{\text {in }} / h$ from 0.1 to 1 only increases $\widehat{\mathcal{E}}_{\text {eff }} 27 \%$, from $26 \mathrm{kPa}$ up to $33 \mathrm{kPa}$, whereas for constant $h_{i n} / h>0.2\left(h_{i n} / h=0.3\right.$ is plotted) an increase of $l_{i n} / l_{2}$ from 0.1 to 1 increases $\widehat{\mathcal{E}}_{\text {eff }}$ with about $270 \%$ from $11 \mathrm{kPa}$ up to $30 \mathrm{kPa}$. Thus the most notable variation of $\widehat{\mathcal{E}}_{\text {eff }}$ for the molded specimens plotted in Fig. 8(a) is predicted to occur for specimens with different inclusion lengths $l_{\text {in }} / l_{2} \in\{0.0,0.2,0.6,1\}$ associated with $\widehat{\mathcal{E}}_{\text {eff }} \in\{10,12,17,30\} \mathrm{kPa}$.

\section{B. Arbitrary layer stacking}

Specimens of type $\mathrm{E}$ with an inclined inclusion $\left(\alpha>0^{\circ}\right)$ in the superficial layer (Fig. 4(e)) or of type $\mathrm{F}$ with a bent inclusion in the muscle layer (Fig. 4(f)) are not serial or parallel stacked with respect to adjacent layers. Instead, their stacking is arbitrary (Arb). However, using the model outlined in section IV A for serial, parallel and combined stacking, two approaches are proposed to model the effective Young's modulus $\widehat{\mathcal{E}}_{\text {eff }}$ for arbitrary stacked layers either based on spatial discretisation along the force direction (section IV B 1) or on geometrical approximation (section IV B 2). A comparison of modelled values with both approaches for molded specimens with arbitrary stacking $E_{0.5}^{0.6}$ and $F_{0.3}$ is provided in section IV B 3.

\section{Discretisation}

The model approach outlined in section IV A is applied to arbitrary stacking by discretising the specimen portion containing the inclusion with equivalent length $l_{e q}$ into multiple shorter layers with discretisation step length $l_{j}$. The inclusion within each discretised layer is then approximated by a rectangle with height $h_{j}$ set either to height $h_{j}^{U}$ of the largest rectangle enveloped within the inclusion or to height $h_{j}^{O}$ of the smallest rectangle enveloping the inclusion in that discretised layer 
as schematically depicted in Fig. 9 for specimens of type E and type F. Consequently, each discretised layer with rectangular inclusion approximation is represented as parallel stacked layers so that $\widehat{\mathcal{E}}_{e f f, j}$ of each homogenised discretised layer is modelled using Eq. (6). The sought $\widehat{\mathcal{E}}_{e f f, j}$ of each discretised layer with length $l_{j}$ is thus underestimated (U) as $\widehat{\mathcal{E}}_{j, U}=\widehat{\mathcal{E}}_{\text {eff }}^{\|}$using height $h_{j}^{U}$ or overestimated $(\mathrm{O})$ as $\widehat{\mathcal{E}}_{j, O}=\widehat{\mathcal{E}}_{e f f}^{\|}$using height $h_{j}^{O}$. Following this discretisation, the equivalent homogenised specimen portion with inclusion and therefore the equivalent homogenised specimen consists of a stack of serial layers, so that $\widehat{\mathcal{E}}_{\text {eff }}$ is modelled using Eq. (5) resulting in overestimation $\widehat{\mathcal{E}}_{O}=\widehat{\mathcal{E}}_{\text {eff }}^{\perp}$ for $\widehat{\mathcal{E}}_{j, O}$ or in underestimation $\widehat{\mathcal{E}}_{U}=\widehat{\mathcal{E}}_{e f f}^{\perp}$ for $\widehat{\mathcal{E}}_{j, U}$. The overall difference $\widehat{\mathcal{E}}_{O}-\widehat{\mathcal{E}}_{U} \geq 0$ for the equivalent homogenised specimen portion with inclusion of length $l_{e q}$ (Fig. 10(c) and Fig. 10(d)) as well as for the equivalent homogenised specimen (Fig. 10(e) and Fig. 10(f)) decreases with discretisation step length $l_{j}$. Consequently, the sought model value $\widehat{\mathcal{E}}_{\text {eff }}$ of the homogenised specimen is found for small enough discretisation step length $l_{j}$. The influence of the discretisation step length $l_{j}$ is illustrated in Fig. 10 for molded specimen $E_{0.5}^{0.6}$ containing an inclined inclusion with $l_{e q}=15.7 \mathrm{~mm}$ (type $\mathrm{E}$ in Fig. 4(f)) and for molded specimen $F_{0.3}$ contain-

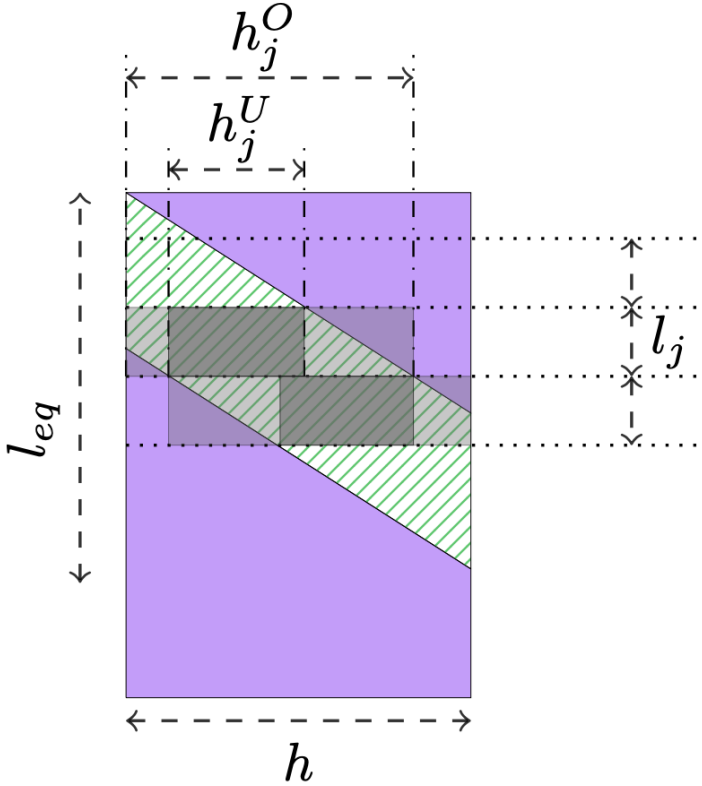

(a) type E, specimen $E_{0.5}^{0.6}$

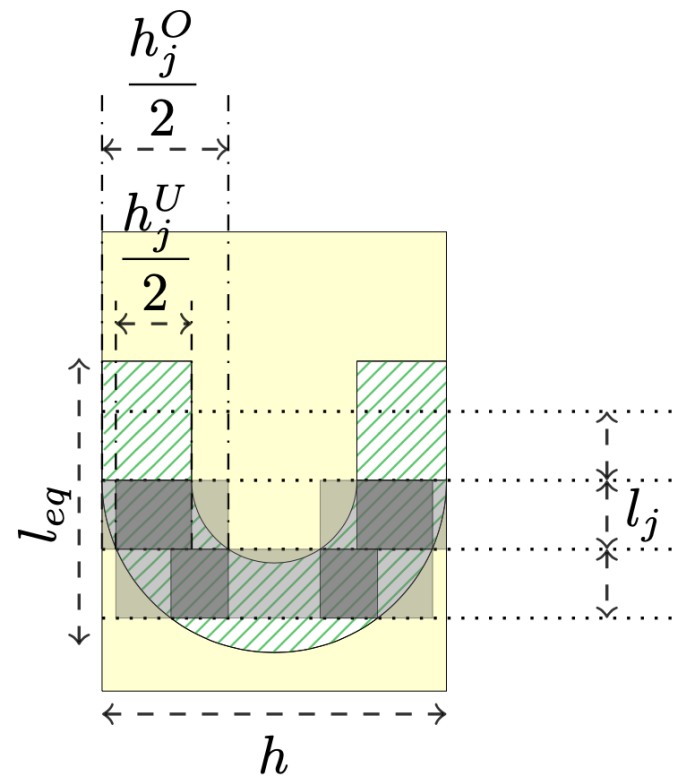

(b) type F, specimen $F_{0.3}$

FIG. 9. Illustration of discretisation along the force direction (step length $l_{j}$ ) for inclusions (striped region) with arbitrary stacking: a) type $\mathrm{E}$ (specimen $E_{0.5}^{0.6}$ ), b) type $\mathrm{F}$ (specimen $F_{0.3}$ ). Rectangular inclusion portion approximations with height $h_{j}^{O}$ (light gray shade) and $h_{j}^{U}$ (dark gray shade) overestimating (O) and underestimating $(\mathrm{U})$ the inclusion, respectively. 
Low-strain inclusion

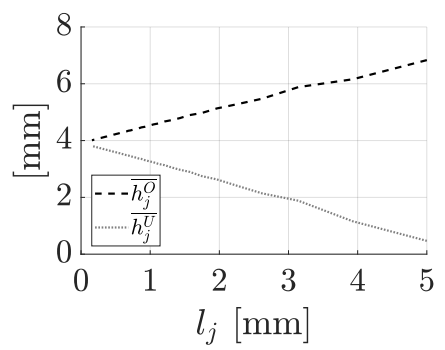

(a) Specimen $E_{0.5}^{0.6}$

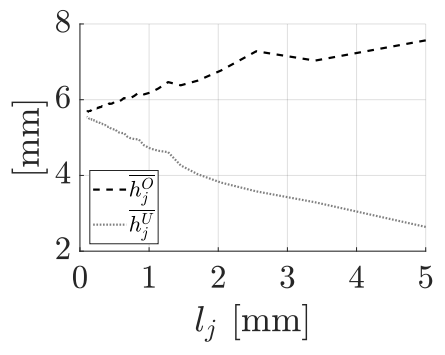

(b) Specimen $F_{0.3}$

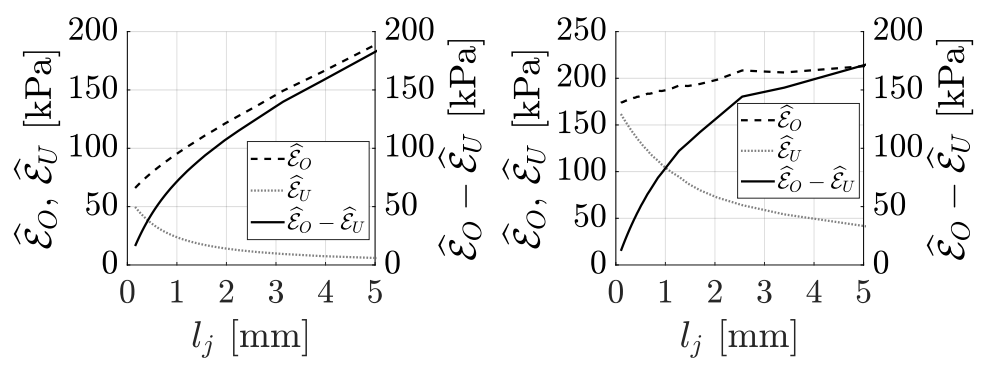

(c) Eq. layer $l_{e q}=16.6 \mathrm{~mm}$ of $E_{0.5}^{0.6}(\mathrm{~d})$ Eq. layer $l_{e q}=10.2 \mathrm{~mm}$ of $F_{0.3}$

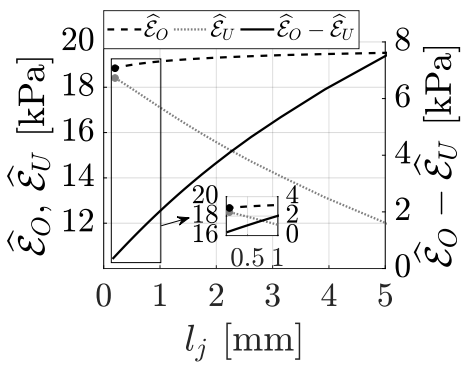

(e) Specimen $E_{0.5}^{0.6}$

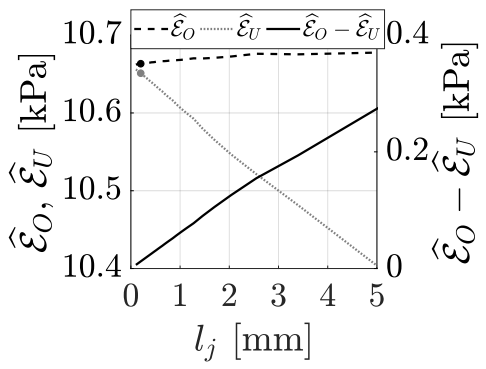

(f) Specimen $F_{0.3}$

FIG. 10. Effect of discretisation step length $l_{j}$ for molded ML specimens $E_{0.5}^{0.6}$ and $F_{0.3}$ on a,b) mean rectangle heights $\overline{h_{j}^{O}}\left(l_{j}\right)$ and $\left.\overline{h_{j}^{U}}\left(l_{j}\right), \mathrm{c}, \mathrm{d}\right)$ modelled effective Young's modulus $\widehat{\mathcal{E}}_{O}\left(l_{j}, h_{j}^{O}\right)$ and $\widehat{\mathcal{E}}_{U}\left(l_{j}, h_{j}^{U}\right)$ and the difference $\widehat{\mathcal{E}}_{O}-\widehat{\mathcal{E}}_{U}$ for the homogenised specimen portion with inclusion of length $l_{e q}$, e,f) modelled effective Young's modulus $\widehat{\mathcal{E}}_{O}\left(l_{j}, h_{j}^{O}\right)$ and $\widehat{\mathcal{E}}_{U}\left(l_{j}, h_{j}^{U}\right)$ and the difference $\widehat{\mathcal{E}}_{O}-\widehat{\mathcal{E}}_{U}$ for the homogenised specimen. Values of $\widehat{\mathcal{E}}_{O, U}$ for $l_{j}=0.2 \mathrm{~mm}$ are indicated $(\bullet)$.

ing a bent inclusion with $l_{e q}=10.2 \mathrm{~mm}$ (type $\mathrm{F}$ in Fig. 4(e)). For these specimens, $\widehat{\mathcal{E}}_{e f f}$ of both the equivalent homogeneous inclusion layer (with length $l_{e q}$ ) and of the specimen is approximated when the discretisation step length $l_{j} \leq 0.2 \mathrm{~mm}$ as $\widehat{\mathcal{E}}_{O}-\widehat{\mathcal{E}}_{U} \leq 0.5 \mathrm{kPa}$ for both homogenised specimens. For $l_{j}=0.2 \mathrm{~mm}$, the discretisation of $l_{e q}$ corresponds to splitting $l_{e q}$ into $78\left(E_{0.5}^{0.6}\right)$ and 51 $\left(F_{0.3}\right)$ equi-length layers. It is seen from Fig. 10(a) and Fig. 10(b) that also the mean of rectangle 
heights for all discretised layers $\overline{h_{j}^{O}}$ and $\overline{h_{j}^{U}}$ converges as $l_{j}$ decreases.

\section{Geometrical approximation}

Besides the discretisation approach outlined in section IV B 1, the quasi-analytical model approach outlined in section IV A can be applied when the inclusion of height $h_{\text {in }}$ and length $l_{\text {in }}$ with arbitrary stacking can be treated as an equivalent beam-shaped inclusion of length $l_{e q}$ and height $h_{e q}$ with serial, parallel or combined layer stacking. The length $l_{e q}$ corresponds to the equivalent length of the inclusion in the force direction as illustrated for type $\mathrm{E}$ and type $\mathrm{F}$ specimens in Fig. 4 and in Fig. 9. The height $h_{e q}$ of the equivalent inclusion is obtained when imposing area conservation and exploiting the model property that neither serial (Eq. (5)) or parallel (Eq. (6)) layer stacks depend on the stack order so that layers in both stacks can be split or permuted. The area conservation condition is defined as $h_{i n} l_{\text {in }}=h_{e q} l_{e q}$ so that

$$
h_{e q}=\frac{h_{i n} l_{i n}}{l_{e q}} .
$$

The dimensions of the equivalent inclusions $h_{e q}$ and $l_{e q}$ can then be expressed in terms of geometrical parameters such as inclusion dimensions $h_{\text {in }}$ and $l_{\text {in }}$.

For specimens of type E, such as $E_{0.5}^{0.6}$ containing an inclined inclusion as depicted in Fig. 4(e), $l_{e q}$ and $h_{e q}$ are expressed as:

$$
\begin{gathered}
l_{e q}=\frac{h_{\text {in }}+h \cos (\alpha)}{\sin (\alpha)}, \\
h_{e q}=\frac{l_{i n} h_{i n} \sin (\alpha)}{h_{\text {in }}+h \cos (\alpha)} .
\end{gathered}
$$

For specimens of type $\mathrm{F}$, such as $F_{0.3}$ containing a bent inclusion as depicted in Fig. 4(f), $l_{e q}$ and $h_{e q}$ are given as:

$$
\begin{gathered}
l_{e q}=\frac{l_{i n}+h}{2}-\frac{\pi\left(h-h_{i n}\right)}{4}, \\
h_{e q}=\frac{4 h_{i n} l_{i n}}{2 l_{i n}+\pi h_{i n}-(\pi-2) h} .
\end{gathered}
$$

Analytical expressions of the geometry of equivalent inclusions, such as Eq. (8) and Eq. (9), are

of interest when considering the influence of geometrical inclusion parameters on $\widehat{\mathcal{E}}_{\text {eff }}$. As an 

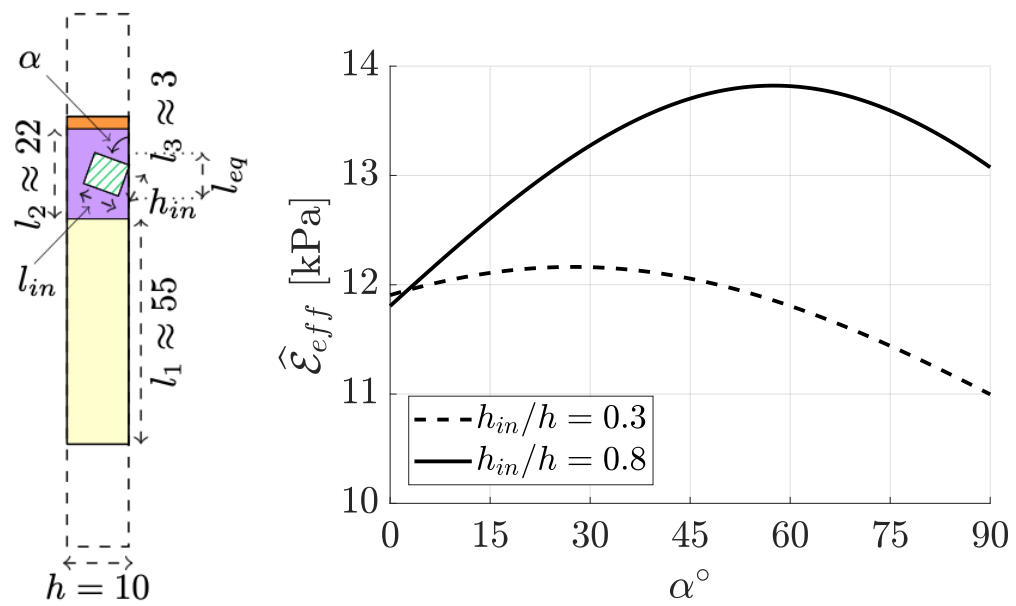

FIG. 11. Modelled $\widehat{\mathcal{E}}_{\text {eff }}$ as a function of inclination angle $\alpha\left(0^{\circ} \leq \alpha \leq 90^{\circ}\right)$ for specimens with an embedded inclusion of diagonal $\sqrt{l_{\text {in }}^{2}+h_{i n}^{2}} \leq h$ as schematically depicted (left). Curves $\widehat{\mathcal{E}}_{\text {eff }}(\alpha)$ are plotted for $l_{\text {in }} / l_{2}=$ 0.2 and either $h_{\text {in }} / h=0.3$ (dashed line) or $h_{\text {in }} / h=0.8$ (full line).

additional example (not molded), expressions of the equivalent length $l_{e q}$ and height $h_{e q}$ of an inclusion with inclination angle $\alpha$ fully embedded in the superficial layer, i.e. with a diagonal shorter than the total specimen height $h$ so that $\sqrt{l_{i n}^{2}+h_{i n}^{2}} \leq h$ as schematically depicted in Fig. 11, are,

$$
\begin{aligned}
& l_{e q}=l_{\text {in }} \cos (\alpha)+h_{\text {in }} \sin (\alpha), \\
& h_{e q}=\frac{l_{\text {in }} h_{\text {in }}}{l_{\text {in }} \cos (\alpha)+h_{\text {in }} \sin (\alpha)} .
\end{aligned}
$$

Modelled $\widehat{\mathcal{E}}_{\text {eff }}$ for two fully embedded inclined inclusions from Table II, with length $l_{\text {in }}=5.0 \mathrm{~mm}$ (or $l_{\text {in }} / l_{2}=0.2$ ) and height $h_{\text {in }} \in\{2.6 \mathrm{~mm}, 7.8 \mathrm{~mm}\}$ (or $h_{\text {in }} / h \in\{0.3,0.8\}$ ), as a function of inclination angle $0^{\circ} \leq \alpha \leq 90^{\circ}$ are plotted in Fig. 11. Although the overall tendency of $\widehat{\mathcal{E}}_{\text {eff }}(\alpha)$ is similar, plotted curves show e.g. that angles associated with the minimum and maximum of the curves depend on the height ratio $h_{\text {in }} / h$.

\section{3. $\widehat{\mathcal{E}}_{\text {eff }}$ of molded specimens with arbitrary stacking}

For the molded specimens with arbitrary stacking, both the discretisation approach (in section IV B 1 with step length $l_{j}=0.2 \mathrm{~mm}$ ) and the geometrical approximation approach (in section IV B 2) result in the sought $\widehat{\mathcal{E}}_{\text {eff }}$ as their difference is less than $0.9 \mathrm{kPa}$ (or $\leq 4.8 \%$ ) for 
Low-strain inclusion

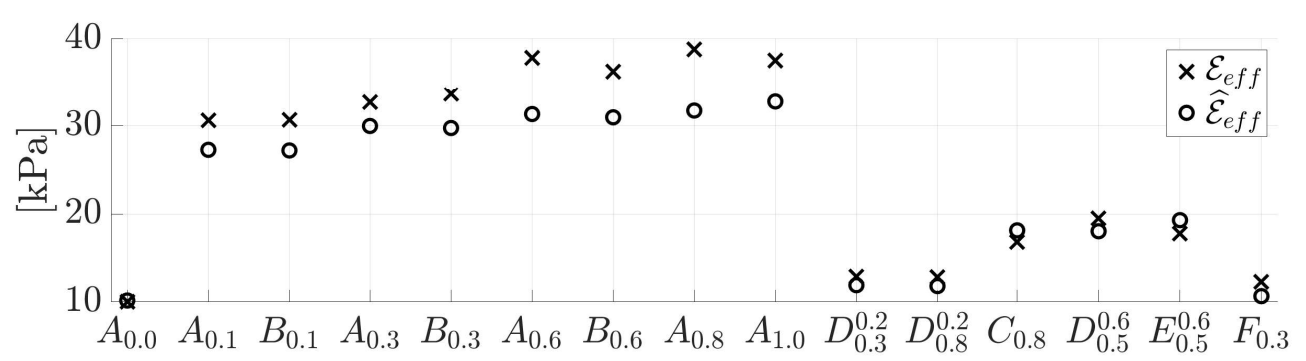

(a)

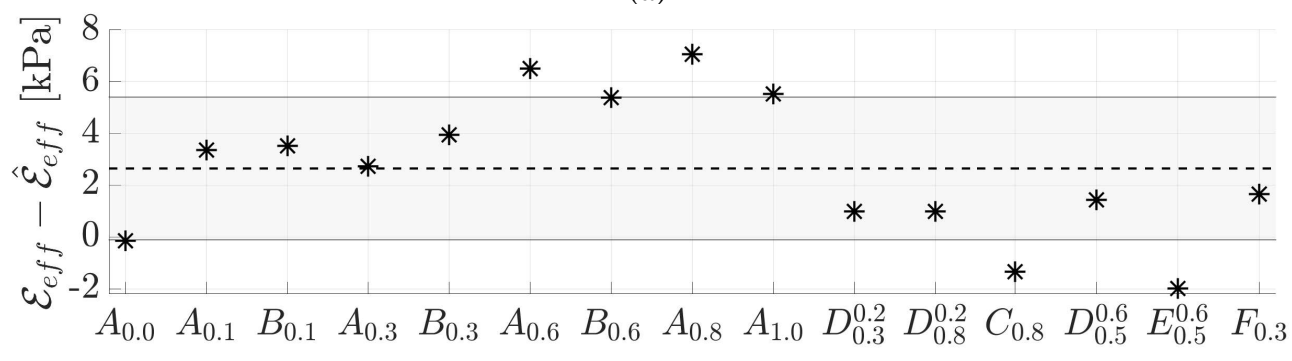

(b)

FIG. 12. Low-strain Young's moduli for molded ML specimens: a) measured $\mathcal{E}_{\text {eff }}(\times)$ and modelled $\widehat{\mathcal{E}}_{\text {eff }}$ (०). b) difference $\mathcal{E}_{e f f}-\widehat{\mathcal{E}}_{\text {eff }}(*)$ with the overall mean (dashed line) and standard deviation (shaded area).

specimen $E_{0.5}^{0.6}$ and less than $0.1 \mathrm{kPa}($ or $\leq 1.0 \%)$ for specimen $F_{0.3}$. Hereafter, $\widehat{\mathcal{E}}_{\text {eff }}$ obtained with the geometrical approximation are reported for specimens with arbitrary stacked inclusions $\left(E_{0.5}^{0.6}\right.$ and $\left.F_{0.3}\right)$ since in this case $\widehat{\mathcal{E}}_{e f f}$ does not depend on the applied discretisation step length $l_{j}$.

\section{MODELLED $\widehat{\mathcal{E}}_{e f f}$ VERSUS MEASURED $\mathcal{E}_{e f f}$}

The model approach outlined in section IV is next used to predict the low-strain $\widehat{\mathcal{E}}_{\text {eff }}$ of each of the 15 molded specimens. Modelled $\widehat{\mathcal{E}}_{\text {eff }}$ and measured $\mathcal{E}_{\text {eff }}$ values are plotted in Fig. 12(a). The difference $\mathcal{E}_{\text {eff }}-\widehat{\mathcal{E}}_{\text {eff }}$ between measured and modelled values is plotted in Fig. 12(b). The absolute value $\left|\mathcal{E}_{e f f}-\widehat{\mathcal{E}}_{e f f}\right|$ for specimens with inclusions corresponds to a model error ranging from $1.0 \mathrm{kPa}$ up to $7.1 \mathrm{kPa}$, which amounts to $7.4 \%$ up to $18.3 \%$ of the measured $\mathcal{E}_{\text {eff }}$. Overall $\mathcal{E}_{\text {eff }}-\widehat{\mathcal{E}}_{\text {eff }}$ of molded specimens is characterised by a mean (and standard deviation) of $2.7(2.7) \mathrm{kPa}$. Compared to the overall model accuracy of 0.0 (2.4) $\mathrm{kPa}$ obtained for two and three layer specimens reported in ${ }^{10}$, the overall model accuracy for specimens with inclusions is thus shifted due to the non-zero mean to the range from $0.0 \mathrm{kPa}$ up to $5.2 \mathrm{kPa}$. The positive non-zero mean of $2.7 \mathrm{kPa}$ indicates that the model tends to underestimate measured values. From Fig. 12 is seen that the underestimation (with $2.7 \mathrm{kPa}$ up to $7.1 \mathrm{kPa}$ ) is associated with specimens 
of type A and type B for which the influence of the inclusion on the Young's modulus is most prominent as their measured effective Young's modulus $\left(\mathcal{E}_{e f f} \geq 30.6 \mathrm{kPa}\right)$ is at least tripled compared to $\mathcal{E}_{\text {eff }}=10.2 \mathrm{kPa}$, measured for the reference specimen without inclusion $A_{0.0}$. Despite this underestimation, the measured $\mathcal{E}_{\text {eff }}$ and modelled $\widehat{\mathcal{E}}_{\text {eff }}$ values exhibit the same tendencies so that the experimental data validate the model approach for all specimen types (including the ones with arbitrary stacking) and also the model properties discussed in section IV such as:

- Comparing measured $\mathcal{E}_{e f f}$ for specimens of type A (inclusion at the side) and type B (inclusion at the center) confirms that the transverse position of the inclusion within the superficial layer does not affect the effective Young's modulus of the specimen as the difference between $\mathcal{E}_{\text {eff }}$ measured for $A_{h_{i n} / h}$ and $B_{h_{\text {in }} / h}$ is less than $1.6 \mathrm{kPa}$ for all three assessed $h_{\text {in }} / h$ ratios $(0.1,0.3,0.6)$.

- Comparing measured $\mathcal{E}_{\text {eff }}$ for specimens of type A (inclusion with $l_{i n} / l_{2}=1$ ) and type D (inclusion with $l_{\text {in }} / l_{2}=0.2$ ) confirms the influence of the inclusion size (length $l_{\text {in }} / l_{2}$ and height $h_{i n} / h$ ratios) on the modelled $\widehat{\mathcal{E}}_{\text {eff }}$ shown in Fig. 8 so that in particular the length of the inclusion in the force direction $\left(l_{i n} / l_{2}\right)$ affects the effective Young's modulus $\mathcal{E}_{e f f}$ for these specimens.

- Comparing measured $\mathcal{E}_{e f f}$ for specimens $A_{0.8}$ (type $\mathrm{A}$, inclusion in the superficial layer) and $C_{0.8}$ (type $\mathrm{C}$, inclusion in both the superficial and the muscle layer) confirms that the influence of the inclusion on measured $\mathcal{E}_{\text {eff }}$ increases with the Young's modulus ratio $\mathcal{E}^{\text {in }} / \mathcal{E}$, which for the molded specimens (Table I) reduces from 74.5 in the superficial layer to 4.6 in the muscle layer.

The influence of the ratio $\mathcal{E}^{I n} / \mathcal{E}$ is further explored using the model. The Young's modulus of the inclusion $\mathcal{E}^{I n}$, and the Young's modulus ratio $\mathcal{E}^{I n} / \mathcal{E}$ of the superficial and muscle layer given in Table I, are scaled as $\gamma \mathcal{E}^{I n}$. The scalar $\gamma$ is varied between $0.2\left(\mathcal{E}^{I n}\right.$ divided by 5$)$ and $5\left(\mathcal{E}^{I n}\right.$ multiplied by 5 ) so that unscaled model values $\widehat{\mathcal{E}}_{\text {eff }}$ for $\mathcal{E}^{I n}$ are obtained for $\gamma=1$. In particular, the scaled Young's modulus of the inclusion $\gamma \mathcal{E}^{\text {In }}$ ranges from $60 \mathrm{kPa}$ up to $1.49 \mathrm{MPa}$ so that the associated Young' modulus ratios $\mathcal{E}^{I n} / \mathcal{E}$ for the superficial layer (ratio from 14.9 up to 372) and for the muscle layer (ratio from 2.6 up to 65) containing the inclusion remain greater than 1 (so an inclusion is embedded in a softer layer). Modelled $\widehat{\mathcal{E}}_{e f f}(\gamma)$ for all 14 composite types with inclusion are plotted in Fig. 13(a). Values $\widehat{\mathcal{E}}_{\text {eff }}$ (circles) for $\gamma=1$ obtained for the molded 
Low-strain inclusion

TABLE III. Influence of scaling inclusions Young's modulus $\mathcal{E}^{I n}$ with scalar $\gamma \in\{0.2,1,5\}$ on modelled $\widehat{\mathcal{E}}_{\text {eff }}$ (in $\mathrm{kPa}$ ) for all 14 composite types with inclusion and the relative maximum difference $\mathcal{D}$ (in $\%$ ) with respect to $\widehat{\mathcal{E}}_{e f f}$ for $\gamma=1$.

\begin{tabular}{l|lllllllllllllll}
\hline$\gamma$ & $A_{0.1}$ & $B_{0.1}$ & $A_{0.3}$ & $B_{0.3}$ & $A_{0.6}$ & $B_{0.6}$ & $A_{0.8}$ & $A_{1.0}$ & $D_{0.3}^{0.2}$ & $D_{0.8}^{0.2}$ & $C_{0.8}$ & $D_{0.5}^{0.6}$ & $E_{0.5}^{0.6}$ & $F_{0.3}$ \\
\hline 0.2 & 18.4 & 18.0 & 22.3 & 22.6 & 26.4 & 26.3 & 27.8 & 29.2 & 11.6 & 11.7 & 16.8 & 16.6 & 17.3 & 10.4 \\
1.0 & 27.3 & 27.2 & 30.0 & 29.8 & 31.3 & 31.0 & 31.7 & 32.8 & 11.9 & 11.8 & 18.1 & 18.0 & 19.3 & 10.7 \\
5.0 & 31.7 & 31.8 & 32.6 & 32.2 & 32.6 & 32.2 & 32.7 & 33.6 & 12.0 & 11.8 & 18.4 & 18.4 & 19.8 & 10.7 \\
\hline $\mathcal{D}$ & 49 & 51 & 34 & 32 & 20 & 19 & 15 & 13 & 3 & 1 & 9 & 10 & 13 & 3 \\
\hline
\end{tabular}

specimens are indicated as a reference. For each composite type, $\widehat{\mathcal{E}}_{e f f}(\gamma)$ increases with $\gamma$. Values of $\widehat{\mathcal{E}}_{e f f}(\gamma)$ for $\gamma \in\{0.2,1.0,5.0\}$ and the relative maximum difference (in $\left.\%\right)$ of $\widehat{\mathcal{E}}_{e f f}(\gamma)$ with

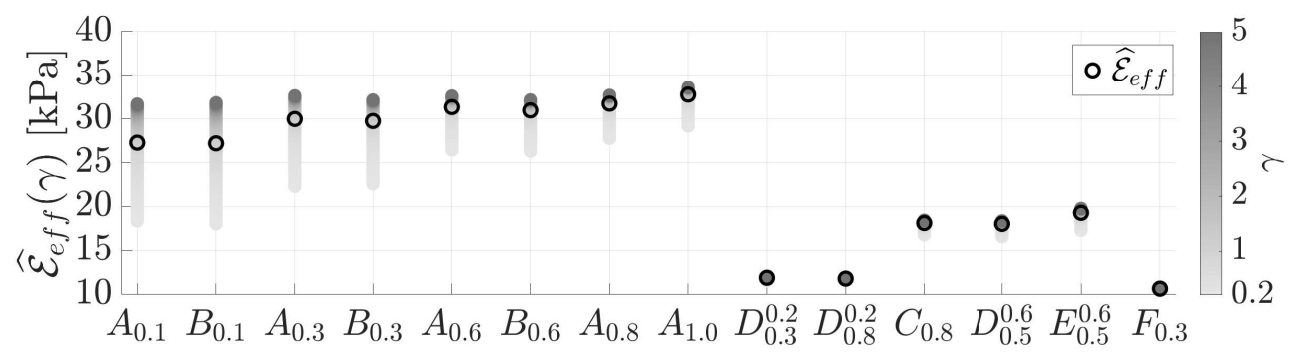

(a) $\widehat{\mathcal{E}}_{\text {eff }}(\gamma)$ (gray scale), $\widehat{\mathcal{E}}_{\text {eff }}$ for $\gamma=1(\circ)$

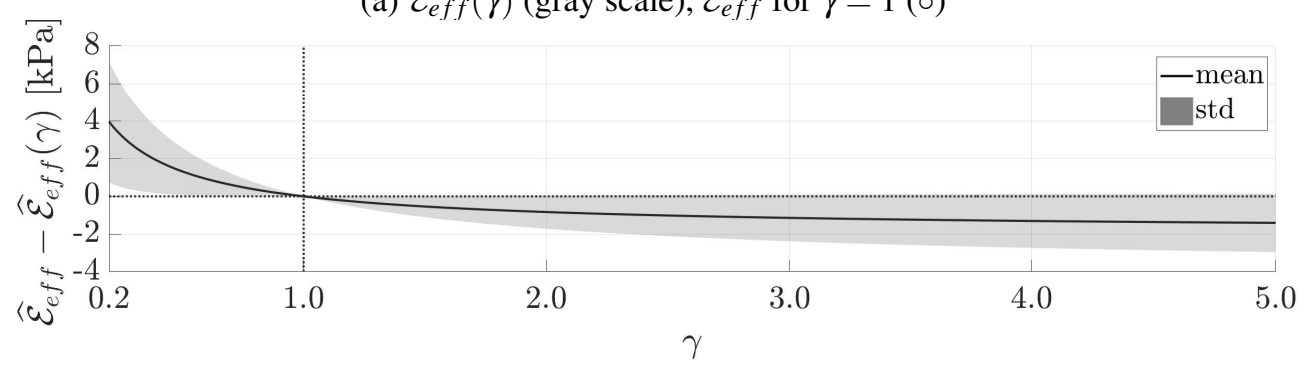

(b) mean and standard deviation of $\widehat{\mathcal{E}}_{\text {eff }}-\widehat{\mathcal{E}}_{e f f}(\gamma)$

FIG. 13. Effect of scaling the Young's modulus of the inclusion $\mathcal{E}^{I n}$ with a scalar $0.2 \leq \gamma \leq 5$ on modelled $\widehat{\mathcal{E}}_{\text {eff }}$ for all $14 \mathrm{ML}$ composite types with inclusion: a) $\widehat{\mathcal{E}}_{\text {eff }}(\gamma)$ for increasing $0.2 \leq \gamma \leq 5$ (gray scale) and $\widehat{\mathcal{E}}_{e f f}$ for $\left.\gamma=1(\mathrm{o}), \mathrm{b}\right)$ overall mean (full line) and standard deviation (std, shaded region) of $\widehat{\mathcal{E}}_{\text {eff }}-\widehat{\mathcal{E}}_{\text {eff }}(\gamma)$. As a reference, dotted lines indicate zero difference (horizontal) and $\gamma=1$ (vertical). 
respect to $\widehat{\mathcal{E}}_{\text {eff }}$ for $\gamma=1$

$$
\mathcal{D}=\frac{\widehat{\mathcal{E}}_{\text {eff }}(5)-\widehat{\mathcal{E}}_{\text {eff }}(0.2)}{\widehat{\mathcal{E}}_{\text {eff }}}
$$

are summarised in Table III. The relative maximum difference $\mathcal{D}$ ranges from $1 \%$ up to $51 \%$. As observed in Fig. 13(a), the influence of scaling the Young's modulus of the inclusion on $\widehat{\mathcal{E}}_{\text {eff }}$ depends on the composite type. The overall (for all composite types) mean and standard deviation (std) of the difference $\widehat{\mathcal{E}}_{e f f}-\widehat{\mathcal{E}}_{e f f}(\gamma)$ as a function of $\gamma$ is plotted in Fig. 13(b). Both the mean and std become zero at $\gamma=1$ for which the difference is zero by definition (since $\widehat{\mathcal{E}}_{\text {eff }}=\widehat{\mathcal{E}}_{\text {eff }}(\gamma=1)$ ). As $\widehat{\mathcal{E}}_{e f f}(\gamma)$ increases with $\gamma$, the overall mean of $\widehat{\mathcal{E}}_{e f f}-\widehat{\mathcal{E}}_{e f f}(\gamma)$ decreases monotonically as $\gamma$ increases. Thus, the overall mean difference is positive for $\gamma<1$ and negative for $\gamma>1$ since $\widehat{\mathcal{E}}_{e f f}>\widehat{\mathcal{E}}_{e f f}(\gamma<1)$ and $\widehat{\mathcal{E}}_{e f f}<\widehat{\mathcal{E}}_{e f f}(\gamma>1)$. The rate at which the overall mean difference decreases slows down with $\gamma$. The model suggests that, for the assessed composite types, $\widehat{\mathcal{E}}_{\text {eff }}$ becomes less sensitive to the exact value of $\mathcal{E}^{I n}$ for large $\mathcal{E}^{I n} / \mathcal{E}$ ratios. The overall standard deviation of $\widehat{\mathcal{E}}_{e f f}-\widehat{\mathcal{E}}_{e f f}(\gamma)$ increases with $|\gamma-1|$, which expresses that the influence of scaling $\mathcal{E}^{\text {In }}$ on $\widehat{\mathcal{E}}_{\text {eff }}$ differs between composite types.

\section{CONCLUSION}

The effective low-strain elastic Young's modulus of silicone ML composites is measured on 15 molded bone-shaped specimens using uni-axial stress testing. A reference specimen is obtained from a three-layer vocal fold anatomical representation of the muscle, superficial and epithelium layers with Young's modulus between $4 \mathrm{kPa}$ and $65 \mathrm{kPa}$. More complex ML composite types with at least 4 layers are obtained by embedding a stiffer (298 kPa) inclusion with variable size, position and stacking in the superficial or/and muscle layer of the reference specimen. Measured effective Young's moduli of all 15 molded ML composite specimens are compared to modelled values describing equivalent homogenised specimens based on the geometry of its layers, Young's moduli and stacking. For ML specimens consisting solely out of serial and/or parallel stacked layers an analytical model approach is applied, which exploits the hypothesis of homogeneous strain for parallel stacked layers and the hypothesis of homogeneous stress for serial stacked layers. A model approach is proposed for specimens for which the inclusion results in arbitrary stacking, first using spatial discretisation along the force direction and then using area conservation to propose a geometrical approximation for inclined or bent inclusions. Modelled effective Young's 
moduli are validated against measured values (from $10 \mathrm{kPa}$ up to $40 \mathrm{kPa}$ ) resulting in an overall model accuracy between $0.0 \mathrm{kPa}$ and $5.2 \mathrm{kPa}$.

The validated quasi-analytical model allows one to explore the influence of its parameters on the predicted effective Young's modulus. Concretely, the influence of the dimensions of an inclusion in the superficial layer and of the influence of scaling the Young's modulus of the inclusion for different ML composite types are discussed. In the first case, the length of the inclusion in the force direction is shown to determine the modelled effective Young's modulus, whereas in the latter case it was found that the rate at which the effective Young's modulus increases with the inclusions slows down, so that eventually for stiff inclusions the exact value of its Young's modulus becomes less important. It is expected, that the proposed model and subsequent model parameter studies are of interest for the a-priori characterisation and design of silicone ML composite vocal fold replicas mimicking the complex ML anatomical vocal fold structure without or with inclusion, as in the case of a structural pathology or abnormality. Therefore, current results are important considering the understanding as well as the mitigation of structural pathologies entraining local vocal fold stiffening and their potential impact on human voice production.

\section{ACKNOWLEDGEMENTS}

This work was partly supported by Full3DTalkingHead project (ANR-20-CE23-0008-03) and a PhD grant from the French Ministry of Education and Research. Authors acknowledge Cristina Pérez Oms for experimental support. The fourth author would like to acknowledge the support from the project FEMVoQ (Ref: PID2020-120441GB-I00) from the Spanish Ministerio de Ciencia e Innovación.

\section{REFERENCES}

${ }^{1}$ C. Rosen, C. Simpson, Operative techniques in laryngology, Springer-Verlag, 2008.

${ }^{2}$ N. Ruty, X. Pelorson, A. Van Hirtum, I. Lopez, A. Hirschberg, An in-vitro setup to test the relevance and the accuracy of low-order models of the vocal folds, J Acoust Soc Am 121 (2007) 479-490. 
${ }^{3}$ J. Lucero, A. Van Hirtum, N. Ruty, J. Cisonni, X. Pelorson, Validation of theoretical models of phonation threshold pressure with data from a vocal fold mechanical replica, J Acoust Soc Am 125 (2009) 632-635.

${ }^{4}$ J. Cisonni, A. Van Hirtum, X. Pelorson, J. Lucero, The influence of geometrical and mechanical input parameters on theoretical models of phonation, Acta Acustica 97 (2011) 291-302.

${ }^{5}$ P. Luizard, X. Pelorson, Threshold of oscillation of a vocal folds replica with unilateral surface growths, J Acoust Soc Am 141 (2017) 3050-3058.

${ }^{6} \mathrm{~J}$. Lucero, X. Pelorson, A. Van Hirtum, Phonation threshold pressure at large asymmetries of the vocal folds, Biomed Sig Proc Control 62 (2020) 102105.

${ }^{7}$ J. Cisonni, A. Van Hirtum, X. Pelorson, J. Willems, Theoretical simulation and experimental validation of inverse quasi one-dimensional steady and unsteady glottal flow models, J Acoust Soc Am 124 (2008) 535-545.

${ }^{8}$ B. Fabre, J. Gilbert, A. Hirschberg, X. Pelorson, Aerocoustics of musical instruments, Ann Rev Fluid Mech 44 (2012) 1-25.

${ }^{9}$ R. Mittal, B. Erath, M. Plesniak, Fluid dynamics of human phonation and speech, Ann Rev Fluid Mech 45 (2013) 437-467.

${ }^{10}$ M. Ahmad, A. Bouvet, X. Pelorson, A. Van Hirtum, Modelling and validation of the elasticity parameters of multi-layer specimens pertinent to silicone vocal fold replicas, Int J Mech Sciences 208 (2021) 106685.

${ }^{11}$ B. Pickup, S. Thomson, Flow-induced vibratory response of idealized versus magnetic resonance imaging-based synthetic vocal fold models, J Acoust Soc Am 128 (2010) 124-129.

${ }^{12}$ P. Murray, S. Thomson, Synthetic, multi-layer, self-oscillating vocal fold model fabrication, J Vis Exp 58 (2011) e3498.

${ }^{13}$ P. Murray, S. Thomson, Vibratory responses of synthetic, self-oscillating vocal fold models, J Acoust Soc Am 132 (2012) 3428-3438.

${ }^{14}$ I. Tokuda, R. Shimamura, Effect of level difference between left and right vocal folds on phonation: Physical experiment and theoretical study, J Acoust Soc Am 142 (2017) 482-492.

${ }^{15}$ R. Shimamura, I. T. Tokuda, Experimental study on level difference between left and right vocal folds, Acoust Sci Technol 38 (2017) 264-267.

${ }^{16}$ A. Bouvet, I. Tokuda, X. Pelorson, A. Van Hirtum, Influence of level difference due to vocal folds angular asymmetry on auto-oscillating replicas, J Acoust Soc Am 147 (2020) 1136-1145. 
${ }^{17}$ A. Bouvet, I. Tokuda, X. Pelorson, A. Van Hirtum, Imaging of auto-oscillating vocal folds replicas with left-right level difference due to angular asymmetry, Biomed Sig Proc Control 63 (2021) 1-12.

${ }^{18}$ A. Van Hirtum, A. Bouvet, I. Tokuda, X. Pelorson, Dynamic vibration mode decomposition of auto-oscillating vocal fold replicas without and with vertical tilting, J Sound Vibr In press (2021) 1-11.

${ }^{19}$ M. Hirano, S. Kurita, T. Nakashima, Vocal Fold Physiology, College Hill Press, 1983, pp. $22-43$.

${ }^{20}$ F. Alipour, I. Titze, Elastic models of vocal fold tissues, J Acoust Soc Am 90 (1991) 1326-1331.

${ }^{21}$ Y. Min, I. Titze, F. Alipour, Stress-strain response of the human vocal ligament, Ann Otol Rhinol Laryngol 104 (1995) 563-569.

${ }^{22}$ R. Chan, M. Fu, L. Young, N. Tirunagari, Relative contributions of collagen and elastin to elasticity of the vocal fold under tension, Ann Biomed. Eng. 35 (2007) 1471-1483.

${ }^{23}$ A. Miri, Mechanical characterization of vocal fold tissue: a review study, J Voice 28 (2014) $657-666$.

${ }^{24}$ D. Chhetri, Z. Zhang, J. Neubauer, Measurement of Young's modulus of vocal folds by indentation, J Voice 25 (2011) 1-7.

${ }^{25}$ Z. Zhang, H. Samajder, J. Long, Biaxial mechanical properties of human vocal fold cover under fold elongation, J Acoust Soc Am 29 (2017) EL356.

${ }^{26}$ J. K. Hansen, S. L. Thibeault, Current understanding and review of the literature: Vocal fold scarring, J Voice 20 (2006) 110-120. doi:10.1016/j.jvoice.2004.12.005.

${ }^{27}$ G. Friedrich, F. G. Dikkers, C. Arens, M. Remacle, M. Hess, A. Giovanni, S. Duflo, A. Hantzakos, V. Bachy, M. Gugatschka, Vocal fold scars: current concepts and future directions. consensus report of the phonosurgery committee of the european laryngological society, Eur Arch Otorhinolaryngology 270 (2013) 2491-2507. doi:10.1007/s00405-013-2498-9.

${ }^{28}$ A. Mattei, J. Magalon, B. Bertrand, C. Philandrianos, J. Véran, A. Giovanni, Cell therapy and scarred vocal folds, Eur Ann Otorhinolaryngology 134 (2017) 339-349. doi: 10.1016/j.anorl.2017.06.006.

${ }^{29}$ A. Bouvet, Experimental and theoretical contribution to the analysis and the modelling of the vocal folds vibration, Ph.D. thesis, Grenoble Alpes University, France, 2019.

${ }^{30} \mathrm{~A}$. Reuss, Berechnung der fließgrenze von mischkristallen auf grund der plastizitätsbedingung für einkristalle, ZAMM 9 (1929) 49-58. 
Low-strain inclusion

${ }^{31} \mathrm{~W}$. Voigt, Ueber die beziehung zwischen den beiden elasticitätsconstanten isotroper körper, Ann Phys 274 (1889) 573-587. 